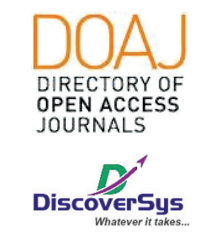

Published by DiscoverSys

\section{Spontaneous intracerebellar haemorrhage and hydrocephalus: A case report}

\author{
Yovi Eko Azhra, ${ }^{1 *}$ M Ihsan Z Tala, ${ }^{2}$ M Deni Nst ${ }^{2}$
}

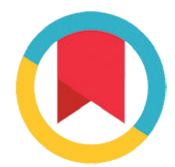

CrossMark

\title{
ABSTRACT
}

Background: Hemorrhagic strokes affect more than 1 million people every year worldwide and are the most frequent type of stroke with high mortality and morbidity rates. Spontaneous Intracerebral Hemorrhage (SICH) often affects the basal ganglia, thalamus, cerebral lobes, pons, and cerebellum.

Case Description: In this report, we present a 63-year-old female with sudden onset loss of consciousness and computed tomography (CT) of head demonstrated an intracranial haemorrhage in the cerebellum with hydrocephalus. The existence of hydrocephalus and the development of chronic hydrocephalus is a problem, awaiting a solution in patients who didn't undergo surgical treatment for cerebellar haemorrhage; however, it can easily be treated effectively by surgical evacuation.

Conclusion: A general practitioner should be able to carry out appropriate initial management to reduce morbidity and mortality.

Keywords: cerebellar haemorrhage, hydrocephalus, stroke.

Cite This Article: Azhra, Y.E., Tala, M.I.Z., Deni, N.S.T.M. 2020. Spontaneous intracerebellar haemorrhage and hydrocephalus: A case report. Intisari Sains Medis 11(2): 805-807. D0I: 10.15562/ism.v11i2.736

${ }^{1}$ General Practitioner, Deli Serdang General Hospital, Lubuk Pakam, Indonesia

${ }^{2}$ Department of Neurosurgery, Faculty of Medicine, Universitas Sumatera Utara, Medan-Indonesia

${ }^{*}$ Correspondence to: Yovi Eko Azhra, General Practitioner, Deli Serdang General Hospital, Lubuk Pakam, Indonesia; yoviieazhra@gmail.com

Received: 2020-03-16 Accepted: 2020-07-11 Published: 2020-08-01

\section{INTRODUCTION}

Hemorrhagic strokes affect more than 1 million people every year worldwide and are the most frequent type of stroke with high mortality and morbidity rates. Uncontrolled hypertension is the most common risk factor for spontaneous hemorrhagic stroke. The incidence of hemorrhagic stroke is higher in Asians because the primary care is limited. Primary prevention with antihypertensive drugs perhaps is the most effective strategy to reduce the burden of hemorrhagic stroke. Although only $10-15 \%$ of all strokes in the United States, hemorrhagic strokes cause disproportionately high levels of morbidity and mortality. In fact, the standard age death rate from hemorrhagic strokes has decreased by more than $25 \%$ over the past 15 years, 3.2 million deaths have been attributed to hemorrhagic strokes compared to 3.3 million deaths due to ischemic stroke in 2013. ${ }^{1}$

Spontaneous Intracerebral Hemorrhage (SICH) often affects the basal ganglia, thalamus, cerebral lobes, pons, and cerebellum. Hypertension, cerebral amyloid angiopathy, and anticoagulation are the leading causes of spontaneous intracerebral haemorrhage. This is caused by damage to hypertension in blood vessels, rupture of aneurysms or arterial malformations, cerebral amyloid angiopathy, altered hemostasis (such as thrombolysis and anticoagulation), hemorrhagic necrosis (such as tumours and infections), or substance abuse (cocaine). Nonvitamin oral anticoagulation $\mathrm{K}$ is associated with lower frequency of spontaneous intracerebral haemorrhage compared to smaller size. ${ }^{2}$

Population-based studies showed the majority of patients with small SICH could easily recover with good medical care. For patients with large SICH, comprehensive multidisciplinary care is essential to minimize morbidity and mortality. Various studies have shown that treating patients with SICH in a special neurological intensive care unit by a special neurocritical care team significantly results in length of stay and rapid mortality. ${ }^{1}$

\section{CASE REPORT}

A 63-year-old female with a past medical history of hypertension was brought to emergency department by her family with complaint of sudden onset loss of consciousness one day prior to admission. Her family stated that she had unstable gait, weakness of both extremities and confusion. There was history of vomiting, spinning-like-headache, without any seizure. History of trauma and alcohol was unremarkable. On arrival, she was unconscious with blood pressure 170/100 mmHg. Her Glasgow Coma Scale (GCS) score was 12 (E:3, M:5, V:4). On neurological examination, her pupils were equal and reactive to light bilaterally with motoric weakness on both extremities (muscle strength: 3/5). Laboratory tests and ECG were normal. Noncontrast Computed Tomography (NCCT) of the head demonstrated an intracranial haemorrhage in the cerebellum with hydrocephalus (Figure 1). 

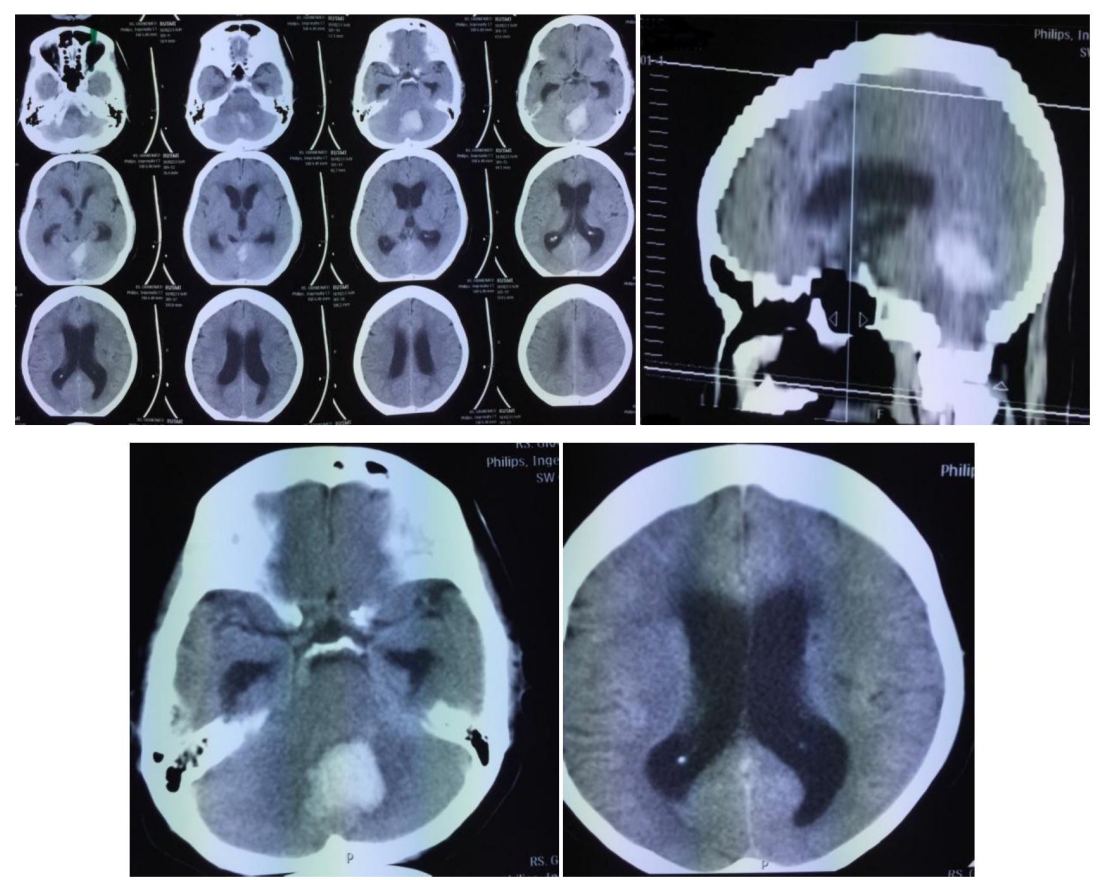

Figure 1 NCCT of The Brain; Head CT shows intracranial haemorrhage in the cerebellum with hydrocephalus

On the ED, she was stabilized and received mannitol intravenous and amlodipine. Her family were immediately informed about the patient's condition and the necessary about transferring to a neurosurgeon in other hospital, however they refused. Then, the patient was hospitalized for 3 days without any improvement. Thereafter, her family requested to bring the patient home despite any risks.

\section{DISCUSSION}

Spontaneous cerebellar hematoma represents 5 to $13 \%$ of all cases of spontaneous intracranial haemorrhage. There have been several risk factors for $\mathrm{ICH}$. Age was known as the most critical unchangeable risk factor for spontaneous ICH. Ariesen et al., Sturgeon et al., and Efstathiou et al., have reported that older age was a major risk factor for ICH. In the present study, the mean age was determined as 62.5 accompanied by the finding that mortality was observed to be higher in patients over the age of 65 . This is in accordance with the literature. A decrease in vessel elasticity with aging makes the vessel wall vulnerable to the effects of hypertension; this may be the hypothetic mechanism for increased risk of cerebral haemorrhage and result in with worse outcomes. ${ }^{3,4}$

The importance of hypertension in spontaneous ICH etiology has been already pointed out in the studies of Sacco, Ariesen et al., and Woo et al. In one study with 152 cases, hypertension was found in $73 \%$ of patients. In another series involving 141 patients, hypertension was determined in $83 \%$ of patients. In this case, the patient was reported with uncontrolled hypertension. This result is remarkable in that it underlines the importance of adequate blood pressure control in the prevention and management of ICH. Recently, antihypertensive treatment of acute cerebral haemorrhage II study group confirmed the efficacy and safety of early, intensive acute antihypertensive treatment, providing evidence of attenuation of hematoma expansion with intensive systolic blood pressure reduction, in subjects with spontaneous $\mathrm{ICH} .{ }^{3}$

One of the symptoms of cerebellar hematoma is acute hydrocephalus, which generally results from the fourth ventricular compression by severe cerebellar hematoma. In this case report, the patient was diagnosed with SICH with hydrocephalus. The existence of hydrocephalus and the development of chronic hydrocephalus is a problem, awaiting a solution in patients who didn't undergo surgical treatment for cerebellar haemorrhage; however, it can easily be treated effectively by surgical evacuation. ${ }^{4}$

\section{CONCLUSION}

Spontaneous intracerebral haemorrhage (ICH), which is defined as spontaneous rupture of the intra-cerebral small vessels following cerebral vessel wall degeneration due to frequent chronic hypertension or rarely to cerebral amyloid angiopathy. The diagnosis of SICH is based on clinical symptoms and supported by blood tests and imaging (CT and Magnetic Resonance Imaging (MRI)).

Mrs. $\mathrm{R}$ was taken to Deli Serdang Regional Hospital in a state of decreased awareness for one day. Initially the patient is on a mild activity and the patient suddenly faints and unconscious. Projectile vomiting $(+)$, headache $(+)$, vertigo $(+)$, limb weakness $(+)$. After physical examination and CT Scan Head without contrast, the patient is diagnosed with hemorrhagic cerebellum stroke with hydrocephalus.

\section{CONFLICT OF INTEREST}

Authors declare there is no conflict of interest in this research.

\section{FUNDING}

The authors received no specific funding for this work. 


\section{AUTHOR CONTRIBUTION}

Each author has a different contribution, Yovi Eko Azhra conceived of the presented idea, developed theory and performed the data. M Ihsan Z Tala and M Deni Nst supervised the project. All authors discussed the results and contributed to the final manuscript.

\section{REFERENCES}

1. Dastur C, Yu W. Current management of spontaneous intracerebral haemorrhage. BMJ. 2017;2(1):21-29.

2. Kumar Garg R, Levine S. Nontraumatic intracerebral haemorrhage. Medlink Clinical Neurology. 2018.
3. Celikbilek A, Goksel BK, Zararsis G, Benli S. Spontaneous intracerebral haemorrhage: A retrospective study of risk factors and outcome in a Turkish population. J Neurosci Rural Pract. 2013;4(3):271-77.

4. Shin D, Woo HJ, Park J. Spontaneous Cerebellar Hemorrhage with the Fourth Ventricular.

5. Haemorrhage: Risk Factors Associated with Ventriculoperitoneal Shunt. J Korean Neurosurg Soc 52. 2012; 320-24.



This work is licensed under a Creative Commons Attribution 